OPEN ACCESS

Edited by:

Péter Poór,

University of Szeged, Hungary

Reviewed by:

Douglas Wendell,

Oakland University, USA

Jeffrey Charles Waller,

Mount Allison University, Canada

*Correspondence:

Xiang Gao

gaoxiang424@163.com

Li Wang

wanglee57@163.com

Specialty section:

This article was submitted to

Plant Physiology,

a section of the journal

Frontiers in Plant Science

Received: 25 October 2016 Accepted: 13 March 2017

Published: 28 March 2017

Citation:

Li Y, Liu X, Cai X, Shan X, Gao R, Yang $S$, Han T, Wang $S$, Wang $L$ and Gao X (2017) Dihydroflavonol

4-Reductase Genes from Freesia hybrida Play Important and Partially Overlapping Roles in the Biosynthesis of Flavonoids. Front. Plant Sci. 8:428. doi: $10.3389 /$ fpls.2017.00428

\section{Dihydroflavonol 4-Reductase Genes from Freesia hybrida Play Important and Partially Overlapping Roles in the Biosynthesis of Flavonoids}

\author{
Yueqing Li, Xingxue Liu, Xinquan Cai, Xiaotong Shan, Ruifang Gao, Song Yang, \\ Taotao Han, Shucai Wang, Li Wang* and Xiang Gao*
}

Key Laboratory of Molecular Epigenetics of MOE, Institute of Genetics and Cytology, Northeast Normal University, Changchun, China

Dihydroflavonol-4-reductase (DFR) is a key enzyme in the reduction of dihydroflavonols to leucoanthocyanidins in both anthocyanin biosynthesis and proanthocyanidin accumulation. In many plant species, it is encoded by a gene family, however, how the different copies evolve either to function in different tissues or at different times or to specialize in the use of different but related substrates needs to be further investigated, especially in monocot plants. In this study, a total of eight putative DFR-like genes were firstly cloned from Freesia hybrida. Phylogenetic analysis showed that they were classified into different branches, and FhDFR1, FhDFR2, and FhDFR3 were clustered into DFR subgroup, whereas others fell into the group with cinnamoyl-CoA reductase (CCR) proteins. Then, the functions of the three FhDFR genes were further characterized. Different spatio-temporal transcription patterns and levels were observed, indicating that the duplicated FhDFR genes might function divergently. After introducing them into Arabidopsis dfr (tt3-1) mutant plants, partial complementation of the loss of cyanidin derivative synthesis was observed, implying that FhDFRs could convert dihydroquercetin to leucocyanidin in planta. Biochemical assays also showed that FhDFR1, FhDFR2, and FhDFR3 could utilize dihydromyricetin to generate leucodelphinidin, while FhDFR2 could also catalyze the formation of leucocyanidin from dihydrocyanidin. On the contrary, neither transgenic nor biochemical analysis demonstrated that FhDFR proteins could reduce dihydrokaempferol to leucopelargonidin. These results were consistent with the freesia flower anthocyanin profiles, among which delphinidin derivatives were predominant, with minor quantities of cyanidin derivatives and undetectable pelargonidin derivatives. Thus, it can be deduced that substrate specificities of DFRs were the determinant for the categories of anthocyanins aglycons accumulated in F. hybrida. Furthermore, we also found that the divergence of the expression patterns for FhDFR genes might be controlled at transcriptional level, as the expression of FhDFR1/FhDFR2 and FhDFR3 was controlled 
by a potential MBW regulatory complex with different activation efficiencies. Therefore, it can be concluded that the DFR-like genes from F. hybrida have diverged during evolution to play partially overlapping roles in the flavonoid biosynthesis, and the results will contribute to the study of evolution of DFR gene families in angiosperms, especially for monocot plants.

Keywords: Freesia hybrida, dihydroflavonol-4-reductase, substrate specificity, transcriptional regulation, functional divergence, cinnamoyl-CoA reductase

\section{INTRODUCTION}

While there is a range of colors found in plants, the predominant color is green. Pigments in plants have several roles, e.g., photosynthesis, signaling, defense or heat exchange. In order to stand out from the predominant green colors of leaves and stems, plants have flowers (and fruits) with many colors and sometimes multiple color patterns (Miller et al., 2011), which are evloved to attract pollinators as visual signals. Floral pigments mainly include carotenoids, betalains, and flavonoids (Tanaka et al., 2008). In most plant species, flower coloration is primarily caused by flavonoids, and the flavonoid family encompasses at least 6000 molecules, chiefly divided into phlobaphenes, aurones, isoflavonoids, flavones, flavonols, flavanols, and anthocyanins (Hichri et al., 2011). Among them, anthocyanins are the most common pigments found in flowers and fruits (Tanaka et al., 2008; Morita et al., 2014) and, thus, are of particular importance.

Anthocyanins are a major class of flavonoids showing bright coloration ranging from blue to orange. Actually, two different types of anthocyanins, 3-hydroxyanthocyanins and 3-deoxyanthocyanins, can be formed in plants (Styles and Ceska, 1975; Halbwirth et al., 2003; Kawahigashi et al., 2016). In contrast to the rare 3-deoxyanthocyanins which have been found only in a few plant species, the ubiquitous 3-hydroxyanthocyanins distribute widely in nature. Thus, the common anthocyanins usually refer to the widely existing 3-hydroxyanthocyanins. Over the past few decades, the biosynthetic pathway of anthocyanins has been well established in plants. They are derived from phenylalanine via the general phenylpropanoid pathway (Figure 1). Actually, the general phenylpropanoid pathway also provides precursors for several branches leading to thousands of compounds, for example, lignins could be synthesized from $\rho$-coumaroyl-CoA by several enzymes containing cinnamoyl-CoA reductase (CCR).

Typically, two classes of genes are confirmed to be involved in the flavonoid pathway: structural genes encoding enzymes that directly participate in the formation of flavonoids, and regulatory genes that control the expression of the structural genes. As shown in Figure 1, one molecule of $\rho$-coumaroyl$\mathrm{CoA}$ and three molecules of malonyl-CoA are catalyzed by chalcone synthase (CHS) to generate naringenin chalcone, which is isomerized to naringenin by chalcone isomerase (CHI). Then, flavanone 3-hydroxylase $(\mathrm{F} 3 \mathrm{H})$ catalyzes the hydroxylation of naringenin to produce dihydrokaempferol (DHK, one hydroxyl group), which is further hydroxylated at the B-ring to form dihydroquercetin (DHQ, two hydroxyl groups) and dihydromyricetin (DHM, 3 hydroxyl groups) by $\mathrm{F}^{\prime} \mathrm{H}$ and $\mathrm{F}^{\prime} 5^{\prime} \mathrm{H}$, respectively. These genes are usually regarded as early biosynthetic genes (EBGs) in flavonoid biosynthetic pathway. Subsequently, dihydroflavonol 4-reductase (DFR) catalyzes dihydroflavonols to leucoanthocyanidins, which are then converted to stable anthocyanins by leucoanthocyanidin di-oxygenase (LDOX) and flavonoid 3-O-glucosyltransferase (3GT) (Holton and Cornish, 1995; Forkmann and Martens, 2001; Jaakola, 2013). And these genes are designated as late biosynthetic genes (LBGs). Comparably, the 3-deoxyanthocyanidin synthesis pathway shared the same early steps, which are consecutively catalyzed by $\mathrm{CHS}$ and $\mathrm{CHI}$. Then the naringenin can be converted to apiforol by flavanone 4-reductase (FNR) or eriodictyol by $\mathrm{F}^{\prime} \mathrm{H}$. Subsequently, eriodictyol can also be catalyzed into luteoforol by FNR (Lo and Nicholson, 1998; Shih et al., 2006; Liu et al., 2010). In addition, other flavonoids can also be synthesized by partially overlapping or competing pathways. For example, the substrate dihydroflavonol of DFR can be catalyzed by flavonol synthase (FLS) to produce flavonols, and leucoanthocyanidins that result from DFR can be converted to proanthocyanidin by leucoanthocyanidin reductase (LAR), while anthocyanidins resulting from LDOX can also be converted to another kind of proanthocyanidin by anthocyanidin reductase (ANR) (Davies et al., 2003; Xie et al., 2003; Xie and Dixon, 2005; Martens et al., 2010; Yoshida et al., 2010). Herein, FNR, DFR, CCR, ANR, and LAR are important components of the NADPHdependent reductase superfamily, which fulfill versatile roles in the biosynthesis of plant secondary metabolites.

Dihydroflavonol-4-reductase is a pivotal oxidoreductase (EC 1.1.1.219) catalyzing the NADPH dependent stereospecific reduction of dihydroflavonols, e.g., dihydrokaempferol, dihydroquercetin and dihydromyricetin, to generate leucopelargonidin (LEUP), leucocyanidin (LEUC), and leucomyricetin (LEUM), respectively (Halbwirth et al., 2006; Petit et al., 2007). The substrate specificity of DFR results in different kinds of anthocyanins, mainly delphinidin derivatives, cyanidin derivatives and pelargonidin derivatives. Mutations in DFR may explain the color transition in some species such as Andean genus Iochroma (Des Marais and Rausher, 2008; Smith and Rausher, 2011; Smith et al., 2013). To our knowledge, various DFR genes have so far been isolated from a wide range of plant species, such as Lotus japonicus (Shimada et al., 2005), Camellia sinensis (Singh et al., 2009), Medicago truncatula (Xie et al., 2004), Malus domestica (Fischer et al., 2003), Pyrus communis (Fischer et al., 2003), Citrus sinensis (Piero et al., 2006), Ipomoea batatas Lam (Wang et al., 2013), Ginkgo biloba (Cheng et al., 2013), and Populus trichocarpa (Huang et al., 2012). However, most of the genes were isolated from dicot plants and few studies 


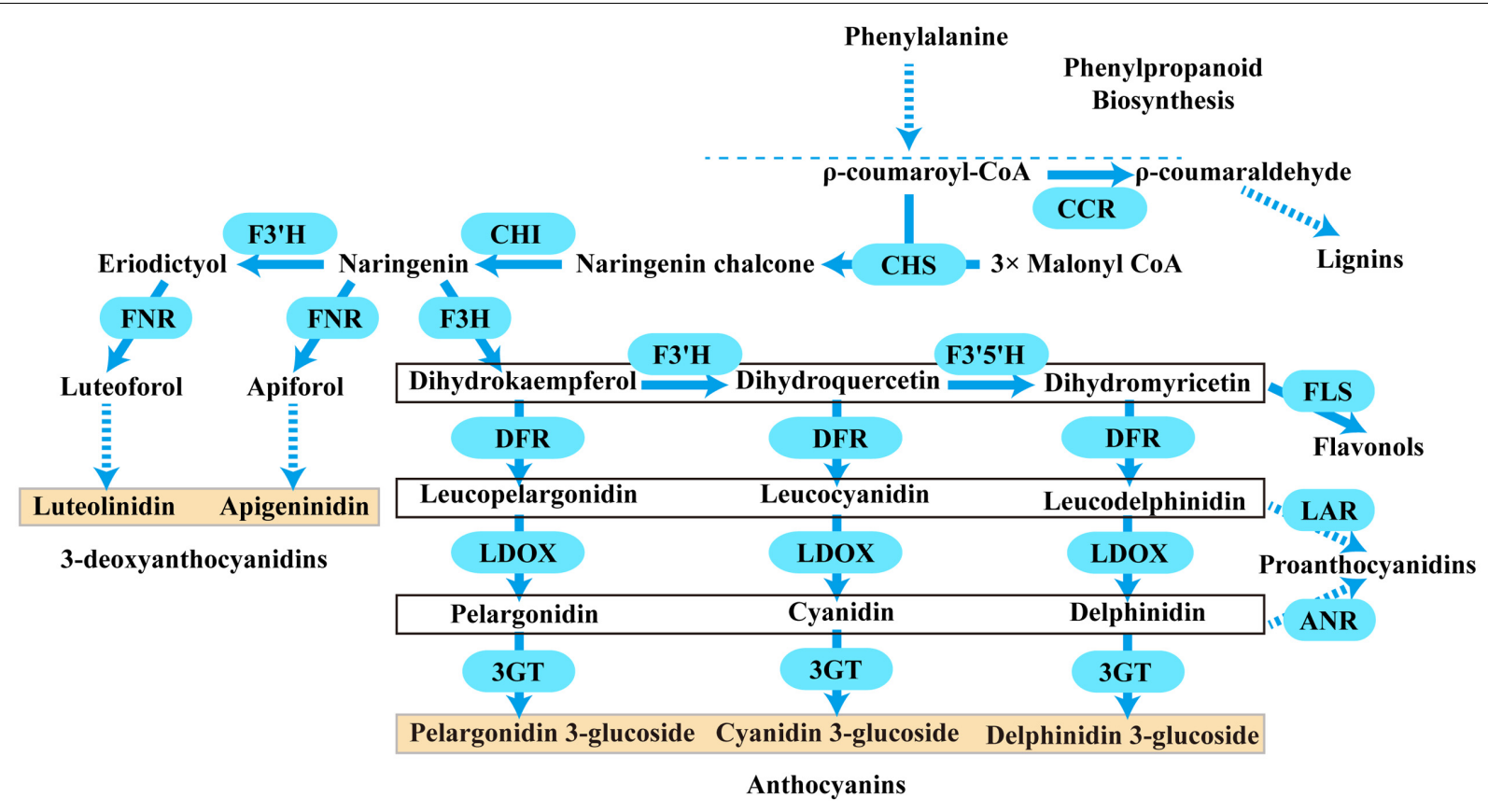

FIGURE 1 | A schematic diagram of the anthocyanins, 3-deoxyanthocyanidins, lignins, flavonols, and proanthocyanidins biosynthetic pathways in plants. Bold arrow indicated one-step process. Dotted arrow indicated multi-step catalytic reaction. CCR, cinnamoyl CoA reductase; CHS, chalcone synthase; CHI, chalcone isomerase; F3H, flavanone 3- hydroxylase; F3'H, flavonoid $3^{\prime}$ - hydroxylase; F3' $5^{\prime} \mathrm{H}$, flavonoid $3^{\prime}, 5^{\prime}$ - hydroxylase; DFR, dihydroflavonol 4- reductase; LDOX, leucoanthocyanidin di-oxygenase; 3GT, flavonoid 3-O-glycosyltransferase; FNR, flavanone 4-reductase; FLS, flavonol synthase; LAR, leucoanthocyanidin reductase; ANR, anthocyanidin reductase.

have focused on the functionally divergence of the members in the DFR gene family, especially in monocot species.

In addition to the structural genes aforementioned, the regulatory mechanism involved in the flavonoid biosynthesis has also been well characterized in plants. Three distinct transcription factor (TF) gene families, containing R2R3 -MYB, basic helix -loop -helix (bHLH) and WD40 repeats (WDRs), comprise a regulatory protein complex (designated as MBW complex) regulating multiple flavonoid metabolisms. At least six MYBs, i.e., PAP1, PAP2, MYB113, MYB114, TT2, MYB5, and three bHLHs, i.e., TT8, GL3, EGL3, and one WD40 (TTG1) regulating the DFR expression in Arabidopsis thaliana have been well elucidated (Baudry et al., 2004; Gonzalez et al., 2008; Pires and Dolan, 2009; Petroni and Tonelli, 2011; Patra et al., 2013; Xu et al., 2014, 2015). To date, some common TF components of the MBW complex regulating DFR expression have been found in maize, petunia, tobacco, and other angiosperms, especially in dicots (Xue et al., 2011; Jaakola, 2013; Xu et al., 2015).

Freesia, a monocotyledonous genus of herbaceous perennial flowering plants in the family Iridaceae, is native to the eastern side of southern Africa, and then widely distributes in the world as a cut flower. The freesia flower colors available include red, pink, yellow, white, blue, lavender, purple, and various bicolors. As a result, it has the potential to be a model system for investigating of flavonoid biosynthesis in monocots, particularly for the flower pigmentation. Our previous studies have confirmed the composition of anthocyanin aglycons, i.e., delphinidin, petunidin, malvinidin, peonidin, and cyanidin, and flavonols, i.e., kaempferol and quercetin derivatives, in Freesia hybrida "Red River ${ }^{\circledR}$," Furthermore, we also found that the accumulation profile for anthocyanins was the opposite of that for flavonols during the flower development process (Sun et al., 2016). In addition, proanthocyanidins were detected (Li et al., 2016) which also showed special accumulation patterns. Therefore, the complicated flavonoid compounds in freesia flowers indicated a sophisticated biosynthetic pathway and transcription regulation network for the flavonoid accumulation. So far, two anthocyanin biosynthetic genes, Fh3GT1 and FhCHS1, as well as two bHLH regulatory genes, FhGL3L and FhTT8L, were isolated and functionally verified (Sui et al., 2011; Sun et al., 2015, 2016; Li et al., 2016). However, no DFR-like genes have been isolated and functionally characterized, which is worthy of further concerns because their particular positions in flavonoid biosynthetic pathway.

In the present study, eight putative DFR-like genes were firstly cloned from flowers of a universal cultivar of F. hybrida, "Red River $^{(\bullet), ~ a n d ~ o n l y ~ t h r e e ~ o f ~ t h e m ~ w e r e ~ p h y l o g e n e t i c a l l y ~ c l u s t e r e d ~}$ into the DFR subgroup, designated as FhDFR1, FhDFR2, and FhDFR3, respectively, which were further functionally characterized. Their temporal and spatial expression profiles were detected and potential roles in planta were investigated through introducing into Arabidopsis $d f r$ (tt3-1) mutant plants. Furthermore, biochemical properties of FhDFR proteins were also determined. Results indicated that dihydroflavonol 4-reductases performed the crucial roles in the anthocyanin 
biosynthesis because of substrate specificities, and their functions were at least partially divergent. As expected, the three FhDFR genes might be controlled by the common MBW complex with diverse regulation efficiencies, because Arabidopsis leaf protoplasts transient expression analysis demonstrated that the earlier characterized FhGL3L and FhTT8L could regulate the expression of FhDFR1/FhDFR2 and FhDFR3 coupled with Arabidopsis endogenous MYB-type TF, AtPAP1, and the promoter of FhDFR3 was activated more extensively. Based on the results aforementioned, a model that elucidated the anthocyanin biosynthesis in F. hybrida was proposed. To our knowledge, this is the first report of the identification of dihydroflavonol 4-reductase gene family in F. hybrida, and the results will not only provide new insights into the flavonoid biosynthesis in monocot plants but also contribute to the study of evolution of DFR gene families in angiosperms.

\section{MATERIALS AND METHODS}

\section{Plant Materials and Growth Conditions}

"Red River ${ }^{\circledR}$," a cultivar of F. hybrida with red flowers, was grown in sandy loam with $\mathrm{pH} 6.5-7.2$ in the greenhouse at $15^{\circ} \mathrm{C}$ with $14 \mathrm{~h} / 10 \mathrm{~h}$ (light/dark) photoperiod. The soil should be kept moist before flower anthesis. For genes isolation and spatio-temporal expression analysis, diverse samples including flowers of five developmental stages with increasing pigmentation intensities and three vegetative tissues, i.e., root, leaf and scape, five flower tissues, i.e., torus, calyx, petal, stamen, and pistil, were collected for RNA extraction as described in our previous studies (Li et al., 2016). All samples were immediately frozen in liquid nitrogen and kept at $-80^{\circ} \mathrm{C}$ prior to total RNA extraction.

Arabidopsis mutant tt3-1 (ABRC stock number: CS84) used for plant transformation was in the Landsberg-0 (Ler) ecotypic background (Shirley et al., 1992), and the seeds were kept at $4^{\circ} \mathrm{C}$ in the dark for 3 days before grown in a growth chamber at $22^{\circ} \mathrm{C}$ with $16 \mathrm{~h} / 8 \mathrm{~h}$ (light/dark) photoperiod. About 5-week-old plants with several mature flowers in the main inflorescence were used for transformation. In order to study the flavonoid accumulation and expression levels of exogenous FhDFR genes from F. hybrida, seeds of wild type, mutant and transgenic plants were surface-sterilized, germinated and cultivated in 1/2 Murashige and Skoog (MS) medium (Murashige and Skoog, 1962) supplemented with 3\% w/v sucrose.

\section{RNA Extraction and cDNA Synthesis}

RNA was extracted from different samples of Freesia or Arabidopsis using OminiPlant RNA Kit (DNase I) (CWBIO) following the manufacturer's instruction. Before cDNA synthesis, RNA was digested with DNase I. cDNA was synthesized in a final reaction volume of $25 \mu \mathrm{l}$ from total RNA (1 $\mu g$ ) using OligodT 15 primers together with M-MLV Reverse Transcriptase (Promega) according to the manufacturer's specifications.

\section{Gene Cloning and Sequence Analysis}

To isolate the candidate DFR-like genes, in situ TBLASTN screen of freesia transcriptomic database, including transcripts from five flower developmental stages and five flower tissues aforementioned, was conducted using Iris $\times$ hollandica DFR (IhDFR, GenBank accession number: BAF93856.1) as probe bait. Sequences obtained were subjected to manual BLASTX search of National Center for Biotechnology Information (NCBI). In order to obtain all the putative candidate DFR genes, we defined the sequence as candidate $D F R$ genes if several hits were named as DFR-like genes in other plant species (Supplementary Table S1). Specific primers were then designed (Supplementary Table S2) to amplify the full length cDNA sequences according to the predicted cDNA sequences. PCR products of appropriate length were cloned into pGEM-Teasy vector (Promega) and then transformed into Escherichia coli JM109 competent cells for sequencing confirmation.

Dihydroflavonol-4-reductase and CCR proteins were retrieved from GenBank for multiple sequence alignment following Clustal Omega algorithm (Sievers et al., 2011). Domains for NADP-binding and substrate-binding were highlighted with different colors. Residues directly influencing the substrate specificity were represented by boxes. For phylogenetic analysis, the full-length amino acid sequences of DFR-like proteins from F. hybrida and other NADPH-dependent reductases in other plant species were aligned with the Clustal Omega using default parameters ${ }^{1}$, and then the alignments were subjected to MEGA version 6 (Tamura et al., 2013) to generate a neighbor-joining tree with bootstrapping (1,000 replicates) analysis and handling gaps with complete deletion.

\section{Quantitative Real-Time PCR Analysis}

In order to study the spatial and temporal expression patterns of FhDFR genes in F. hybrida, specific quantitative real-time PCR primers were designed. Transcript levels were analyzed using SYBR Master Mix (TOYOBO, Japan) and a StepOnePlus RealTime PCR System (Applied Biosystems, USA). All biological replicates were analyzed in triplicate. PCR parameters were set as previously reported (Li et al., 2016). Briefly, a total volume of $10 \mu \mathrm{l}$ of reaction mixture containing $5 \mu \mathrm{l}$ of $2 \times$ Master Mix, $0.5 \mu \mathrm{M}$ of each primer, and $1 \mu \mathrm{lcDNA}$ were analyzed using the following cycling conditions: $95^{\circ} \mathrm{C}$ for $60 \mathrm{~s}$, followed by 40 cycles of $95^{\circ} \mathrm{C}$ for $5 \mathrm{~s}$ and $60^{\circ} \mathrm{C}$ for $60 \mathrm{~s}$. Real-time PCR reactions were normalized to the $\mathrm{Ct}$ values for freesia $18 \mathrm{~S} r \mathrm{RNA}$. The relative expression levels of the target genes were calculated using the formula $2^{-\Delta \Delta C T}$ (Livak and Schmittgen, 2001).

\section{Plant Transformation}

All the three FhDFR genes digested by BamH I and Sac I were cloned into pBI121 vector harboring the CaMV 35S constitutive promoter and confirmed by sequencing. The constructs were then transformed to Agrobacterium tumefaciens strain GV3101 using a freeze-thaw method. About 5 6-week old Arabidopsis plants with a few mature flowers on the main stems were transformed through the floral dip method (Clough and Bent,

\footnotetext{
${ }^{1}$ http://www.ebi.ac.uk/Tools/msa/clustalo/
} 
1998). T1 seeds were selected on $1 / 2$ MS medium containing $50 \mathrm{mg} \mathrm{L}{ }^{-1}$ kanamycin and transferred to soil to set $\mathrm{T} 2$ seeds. The T2 seeds were then cultured on 1/2 MS medium containing $25 \mathrm{mg} \mathrm{L}^{-1}$ kanamycin and 3\% sucrose. After 1 week of culture on anthocyanin biosynthetic gene induction media, transgenic lines were subjected to evaluate expression level of exogenous FhDFR genes and flavonoid accumulation. The Arabidopsis actin gene was used as internal control gene when detecting the FhDFRs expression levels in transformed mutant lines (Penninckx et al., 1997).

\section{Measurement of Flavonol and Anthocyanin Contents in Arabidopsis}

Total anthocyanin content and the amount of flavonol were determined in both wild type, mutants and transgenic plants according to previously described methods (Sun et al., 2016). Briefly, 1-week-old Arabidopsis seedlings cultured on 1/2 MS medium with $3 \% \mathrm{w} / \mathrm{v}$ sucrose were ground in liquid nitrogen and submerged in $1 \mathrm{~mL} \mathrm{H}_{2} \mathrm{O}: \mathrm{MeOH}: \mathrm{HCl}(75 / 24 / 1 \mathrm{v} / \mathrm{v} / \mathrm{v})$. Extracts were centrifuged and the supernatant was collected. Chromatographic analysis was carried out on a Shimadzu HPLC system equipped with an autosampler with a $20 \mu$ l loop, a LC6AD HPLC Pump and an ACCHROM XUnion C18 column $(250 \mathrm{~mm} \times 4.6 \mathrm{~mm}, 5 \mu \mathrm{m})$. The column was eluted with solvent systems $\mathrm{A}$ ( $5 \%$ formic acid in $\mathrm{H}_{2} \mathrm{O}$ ) and $\mathrm{B}$ (methanol) under the following conditions: $0-10 \mathrm{~min}, 14-17 \% \mathrm{~B} ; 10-35 \mathrm{~min}, 17-23 \%$ B; 35-60min, $23-47 \%$ B; 60-67 min, 47-14\% B; 67-70 min, $14 \%$ $B$ with a flow rate of $1 \mathrm{ml} \mathrm{min}{ }^{-1}$. Detection was monitored at 520 and $360 \mathrm{~nm}$ for anthocyanins and flavonols, respectively.

Qualitative analysis of anthocyanin derivatives were conducted by using high performance liquid chromatography (HPLC)-electrospray ionization (ESI)-tandem mass spectrometry (MS) analysis as described earlier (Sun et al., 2016). Briefly, API2000 mass spectrometer (AB Sciex) and SPD20AV UV/VIS Detector (Shimadzu, Kyoto, Japan) were equipped with an ESI source. Ion Trap source parameters in positive mode were as follows: ESI source voltage, $4.5 \mathrm{kV}$; gas (N2) temperature, $450^{\circ} \mathrm{C}$; declustering potential, $\mathrm{C} 80 \mathrm{~V}$; entrance potential, $10 \mathrm{~V}$; and scan range, $\mathrm{m} / \mathrm{z}$ 100-1000 units. Metabolites were identified by their retention times, mass spectra, and product ion spectra in comparison with the data of authentic standards.

\section{Heterologous Expression of FhDFR Proteins in Escherichia coli and In vitro Enzyme Assay}

Heterologous expression of FhDFR genes, which were determined to restore the phenotype of Arabidopsis mutant $t t 3-1$, and enzyme assay was carried out following the previously described methods (Sun et al., 2015, 2016). Briefly, FhDFR genes were subcloned into the $p E T 28 a$ vector and expressed as $\mathrm{N}$-terminal His-tagged proteins. An empty vector and vectors harboring different FhDFR cDNAs were used for transformation of E. coli strain BL21 (DE3). Then the transformants were pre-cultured at $37^{\circ} \mathrm{C}$ overnight in LB media containing $50 \mathrm{mg}$ $\mathrm{L}^{-1}$ kanamycin. The preculture was then transferred to fresh LB media containing $50 \mathrm{mg} \mathrm{L}{ }^{-1}$ kanamycin and cultured at $37^{\circ} \mathrm{C}$ until an $A_{600}$ of 0.6 was reached. Recombinant proteins were then induced by adding $0.2 \mathrm{mM}$ isopropyl-b-d-thiogalactopyranoside (IPTG), and the optimal induction condition was $28 \mathrm{~h}$ and $15^{\circ} \mathrm{C}$ for FhDFR1 and FhDFR2, $28 \mathrm{~h}$ and $20^{\circ} \mathrm{C}$ for FhDFR3, respectively. After induction, the cells were harvested by centrifugation, resuspended in phosphate-buffered saline (PBS, $\mathrm{pH} 7.4$ ), and disrupted by sonication. After centrifugation at $13,225 \mathrm{~g}$ for $20 \mathrm{~min}$, the supernatant containing crude proteins was then applied to $3 \mathrm{ml}$ PBS-equilibrated Ni Sepharose column (GE Healthcare). The column was then washed to remove non-specifically bound proteins using gradient imidazole in PBS. The purified proteins were eluted from the column using $100 \mathrm{mM}$ imidazole in PBS. Eluted FhDFR proteins were desalted in PBS to remove the imidazole at $4^{\circ} \mathrm{C}$. The desalted FhDFR proteins were then concentrated and assessed by SDS-PAGE with Coomassie Brilliant Blue staining (Supplementary Figure S1). After that, their concentrations were detected by NanoDrop 1000 (Thermo scientific) Spectrophotometer before enzymatic assays.

Substrate specificities of FhDFR proteins were carried out as described by Cheng et al. (2013). Shortly, DHK, DHM, and DHQ bought from Sigma were dissolved in methanol at $10 \mathrm{mg} / \mathrm{mL}$. A $500 \mu \mathrm{l}$ reaction mixture consisting of $370 \mu \mathrm{L}$ of $100 \mathrm{mM}$ Tris$\mathrm{HCl}$ buffer (pH7.0), $70 \mu \mathrm{L}$ of $0.5 \mathrm{mg} / \mathrm{ml}$ FhDFR enzyme extract, $10 \mu \mathrm{L}$ of substrate, and $50 \mu \mathrm{L}$ of $20 \mathrm{mM}$ NADPH was kept at $30^{\circ} \mathrm{C}$ for $30 \mathrm{~min} .20 \mu \mathrm{L}$ of reaction solution was resolved on an ACCHROM XUnion C18 column. The column was eluted with solvent systems $\mathrm{A}\left(1 \% \mathrm{H}_{3} \mathrm{PO}_{4}\right.$ in water) and $\mathrm{B}$ (methanol) under the following conditions: $0 \mathrm{~min}, 15 \% \mathrm{~B} ; 0-20 \mathrm{~min}, 15-60 \% \mathrm{~B}$; 20-30min, $60-15 \%$ B. Detection was monitored at $280 \mathrm{~nm}$, the maximum absorbance wavelength for most of the substrates and products.

\section{DNA Extraction and Plasmid DNA Preparation Used in Arabidopsis Leaf Protoplast Transfection Assay}

DNA was extracted from freesia flowers using NuClean Plant Genomic DNA Kit (CWBIO) according to the manufacturer's instruction. Promoters of FhDFR1/FhDFR2 and FhDFR3 were cloned using Genome Walking Kit (TaKaRa) following the instructions. The -1466 bp of FhDFR1/FhDFR2 and -1132 bp of FhDFR3 from the initiation condon "ATG" were amplified as promoters and cloned into Pst I and Sac I digested AtDFRpro:GUS construct to generate FhDFR1/FhDFR2-pro:GUS and FhDFR3-pro:GUS, respectively. All the other constructs used for protoplasts transfection have been described previously ( $\mathrm{Li}$ et al., 2016). All the plasmids were prepared using the EndoFree Plasmid Maxi Kit (CWBIO) following the manufacturer's instructions.

\section{Protoplast Isolation, Transfection and GUS Activity Assay}

Protoplast isolation, transfection and GUS activity assays were performed as described previously (Wang and Chen, 2014; Zhou et al., 2014). Briefly, 3 to 4 -week-old Col wild type Arabidopsis rosette leaves were collected and used to isolate protoplasts. FhDFR1/FhDFR2-pro:GUS and FhDFR3-pro:GUS 
constructs were transformed with different effecter plasmids into protoplasts. A $10 \mu \mathrm{g}$ aliquot of each plasmid was used in transfection assays. After 20-22 $\mathrm{h}$ incubation at room temperature in the dark, the protoplasts were lysed and incubated with 4 -methyl-umbelliferyl- $\beta$-D-glucuronide (MUG) assay solution at $37^{\circ} \mathrm{C}$ for $50 \mathrm{~min}$. GUS activities were measured using a Synergy ${ }^{\mathrm{TM}}$ HT microplate reader (BioTEK). The assays were repeated three times with three biological replicates.

\section{RESULTS}

\section{Isolation and Characterization of DFR-Like Genes from Freesia hybrida}

Amino acid sequence of IhDFR was used as bait probe during in situ TBLASTN search of transcriptomic database of F. hybrida.
Consequently, eight putative sequences encoding Rossmannfold $\mathrm{NAD}(\mathrm{P})(+)$-binding proteins were isolated and predicted as flavonoid reductases (FRs) genes which might be the DFRlike genes in freesia. Among them, three genes were named as FhDFR1, FhDFR2, and FhDFR3, which were most likely to be bona fide DFR genes, because they encoded proteins sharing 65, 66, and 65\% identities to Arabidopsis DFR, and 79, 79, and 77\% identities to Iris $\times$ hollandica DFR, respectively (Supplementary Table S1). In contrast, other five genes might encode CCRlike proteins as the best hits of manual BLASTX search were CCRs from other plant species (Supplementary Table S1). Thus, they were tentatively designated as FhCCR1, FhCCR2, FhCCR3, FhCCR4, and FhCCR5, respectively.

Moreover, FhDFR1 and FhDFR2 shared the highest nucleotide identity of 95\%, in comparison to FhDFR1, FhDFR2 showed nine exchanges and the last change from "CGA" to "TGA" resulted in a premature stop codon. And this resulted in a substitution of four
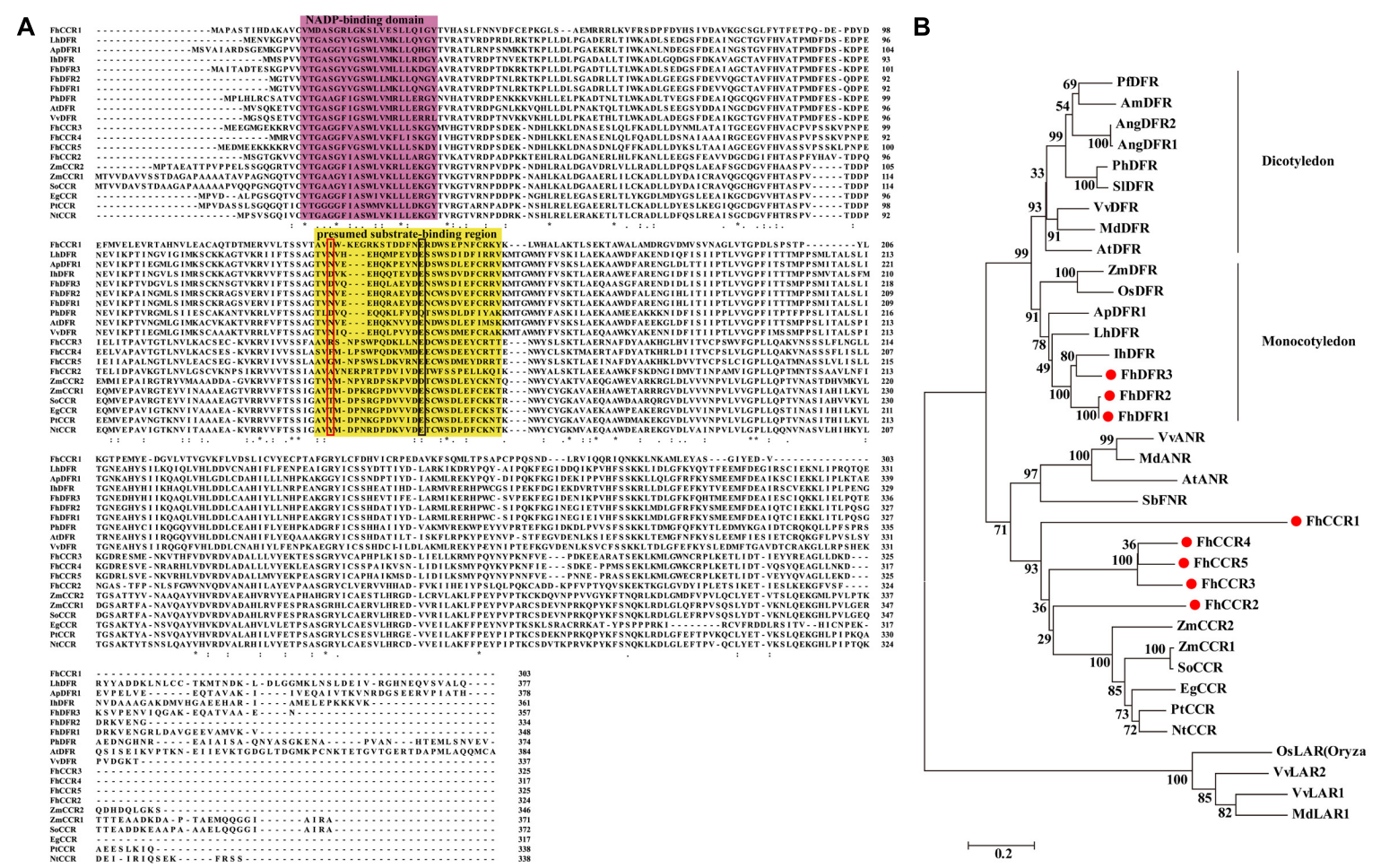

FIGURE 2 | Alignment of amino acid sequences and phylogenetic analysis of the DFR-like and CCR-like proteins in Freesia hybrida with proteins from other species. (A) Multiple alignment of freesia proteins with DFR and CCR proteins from other species. Numbers indicated the position of the last amino acid in each line of the proteins. *, identical amino acids; : or ., similar amino acids. The putative NADP binding site and presumed substrate-binding region were shaded in different colors, respectively. Substrate specificity is associated with particularly aa134 and aa145 in the red and black boxes (Johnson et al., 2001). (B) Phylogenetic relationships between freesia DFR-like and CCR-like proteins and other NADPH-dependent reductase proteins from other plant species. Phylogenetic tree was constructed using the neighbor-joining method by the MEGA6 software. The reliability of the trees was tested using a bootstrapping method with 1000 replicates. Numbers indicate bootstrap values for 1000 replicates. Freesia proteins were indicated with red circles. The GenBank accession numbers of the protein sequences used are as follows: Iris $\times$ hollandica IhDFR (BAF93856.1); Agapanthus praecox ApDFR1 (AB099529.1); Arabidopsis thaliana AtDFR (AB033294), AtANR (Q9SEV0.2); Lilium hybrid LhDFR (AB058641); Petunia $\times$ hybrida PhDFR (AF233639); Vitis vinifera VvDFR (X75964), VvLAR1 (AAZ82410), VvLAR2 (AAZ82411), VvANR (BAD89742); Angelonia angustifolia AngDFR1 (KJ817183), AngDFR2 (KF285561); Zea mays ZmDFR (Y16040), ZmCCR1 (Y13734), ZmCCR2 (Y15069); Oryza sativa OsLAR (CAl56328.1), OsDFR (AB003495); Perilla frutescens PfDFR (AB002817); Malus domestica MdDFR (AAO39816), MdLAR1 (AAZ79364.1), MdANR (AEL79861.1); Solanum lycopersicum SIDFR (CAA79154.1); Antirrhinum majus AmDFR (X15536); Sorghum bicolor SbFNR (BAU68557.1); Eucalyptus gunnii EgCCR (X97433); Populus trichocarpa $\times$ P. deltoides PtCCR (A47097); Nicotiana tabacum NtCCR (A47101); Saccharum officinarum SoCCR (AJ231134); Freesia hybrida FhDFR1 (KU132393), FhDFR2 (KU132389), FhDFR3 (KU132390), FhCCR1 (KU132391), FhCCR2 (KU132392), FhCCR3 (KU132388), FhCCR4 (KU132394), FhCCR5 (KU132395). 


\section{A}

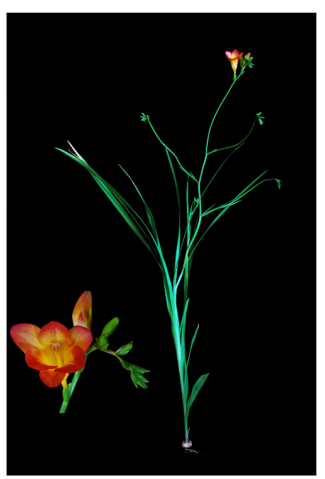

C

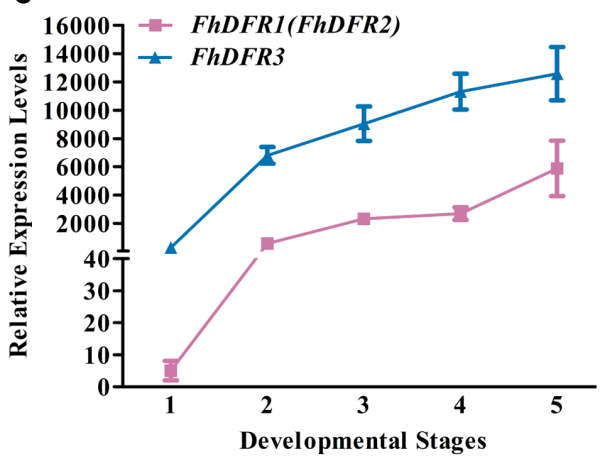

B
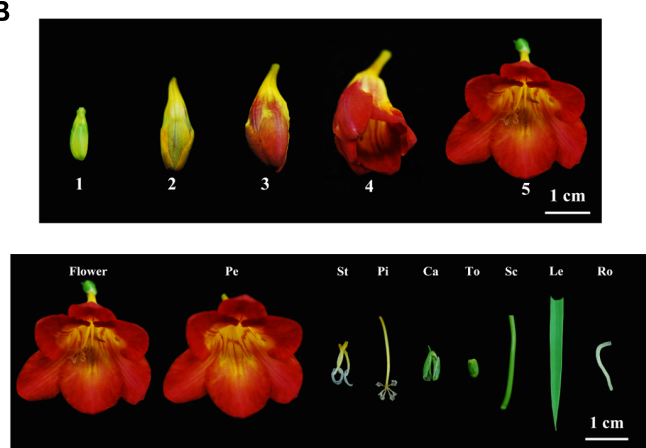

D

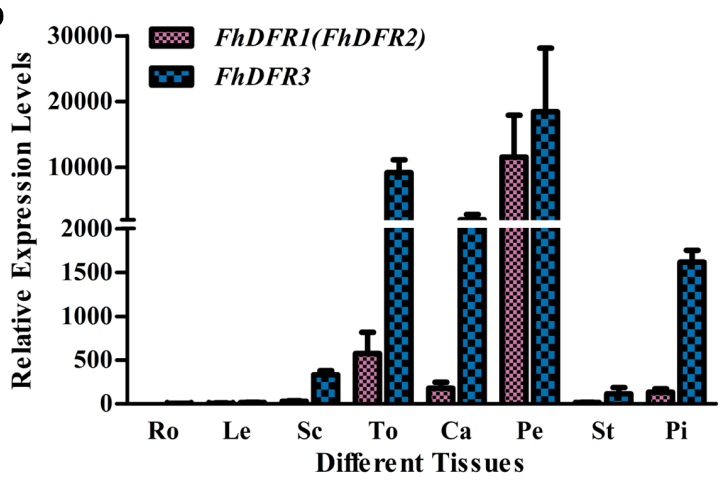

FIGURE 3 | Expression profiles of FhDFR genes in F. hybrida. (A) Phenotypic trait of Red River ${ }^{\circledR}$, a cultivar of $F$. hybrida. (B) Five developmental stages of flowers and different tissues. Stage $1<10 \mathrm{~mm}$ long with unpigmented buds; Stage 2 10-20 mm long with slightly pigmented buds; Stage 3 20-30 mm long with pigmented buds; Stage 4 fully pigmented flowers before complete opening; Stage 5 fully opened flowers. Pe, petals; St, stamens; Pi, pistils; Ca, calyxes; To, toruses; Sc, scapes; Le, leaves; Ro, roots. The developmental stages and tissues were selected as described earlier. (C,D) Expression profile of FhDFR genes in flowers at different developmental stages and in different tissues, respectively. Data represent means \pm SD of three biological replicates.

amino acids and a deletion of 14 amino acids at the C-terminus (Figure 2A). Sequence alignment with a number of NADPHdependent reductases showed that the $\mathrm{N}$-terminus regions of eight F. hybrida proteins contained putative NADP-binding region and substrate-binding region which was composed of 26 amino acid residues (Lacombe et al., 1997; Johnson et al., 2001). Moreover, results here also suggested that FhDFR1, FhDFR2, and FhDFR3 were more similar to the identified DFRs in other species, whereas other five proteins tended to be CCRs.

To further investigate the amino acid sequence homology of the eight freesia proteins to other known DFRs and CCRs, as well as other NADPH-dependent reductases such as LAR, ANR, and FNR, a phylogenetic tree was generated by the neighbor-joining method, and the results showed that DFRs from monocots and eudicots were clearly classified into different branches (Figure 2B), DFR-like proteins, including FhDFR1, FhDFR2, and FhDFR3, clustered within a subgroup containing proteins from monocot plant species and were most similar to Iris $\times$ hollandica DFR (Katsumoto et al., 2007), indicating that these three DFR-like proteins might participate in the catalyzing of the NADPH-dependent reduction of 2R, 3R-trans-dihydroflavonols to leucoanthocyanidins in the flavonoid biosynthetic pathway. Furthermore, other five proteins from F. hybrida clustered independently outside the core DFR branch and fell into a subclade containing CCRs in other species, implying that they might be members of NADPH dependent CCR family.

\section{The Expression of FhDFR Genes Showed Different Correlations with Flavonoid Accumulation in Flower Developmental Process and Plant Tissues}

Freesia hybrida, as a beautiful perennial herb, sends up a tuft of narrow leaves $10-30 \mathrm{~cm}$ long, and a sparsely branched stem $10-40 \mathrm{~cm}$ tall bearing a few leaves and a loose one-sided spike of flowers with six tepals (Figure 3A). To examine whether the expression patterns of the three potential FhDFR genes in flower developmental stages and various tissues coincided with anthocyanin and/or proanthocyanidin accumulation in F. hybrida, quantitative real-time PCR was performed to investigate their expression levels temporally and spatially using gene specific primers. It was worth mentioning that as FhDFR1 and FhDFR2 showing high homogeneity, no specific primer sets could distinguish the two sequences. FhDFR1 and FhDFR2 were evaluated together in expression pattern analysis. Developmental stages of the flower of $F$. hybrida were described earlier as follows (Figure 3B): Stage 1, flower buds with non-pigmented tepals; Stage 2, flower buds with pale-red tepals; Stage 3, flower buds with red tepals; Stage 4, flower bud just after anthesis; and Stage 5, 
A
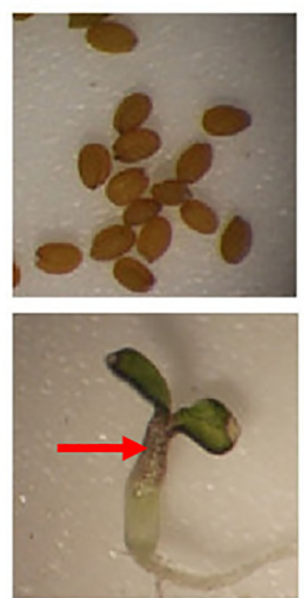

B

Wild type
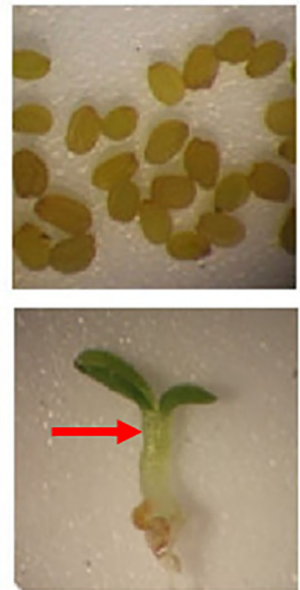

Mutant
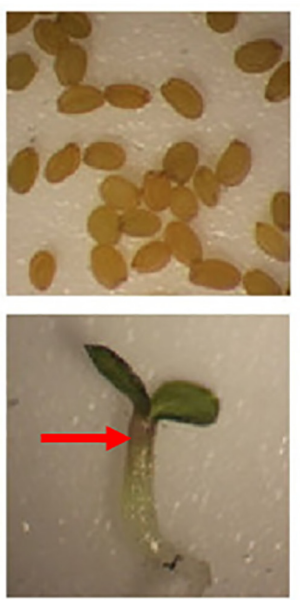

FhDFR 1
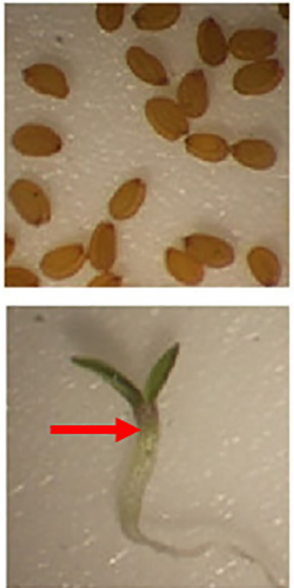

FhDFR2

Transgenic
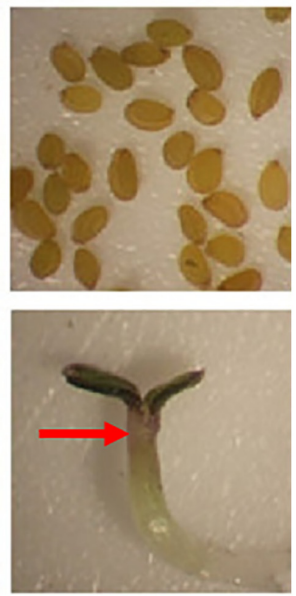

FhDFR3

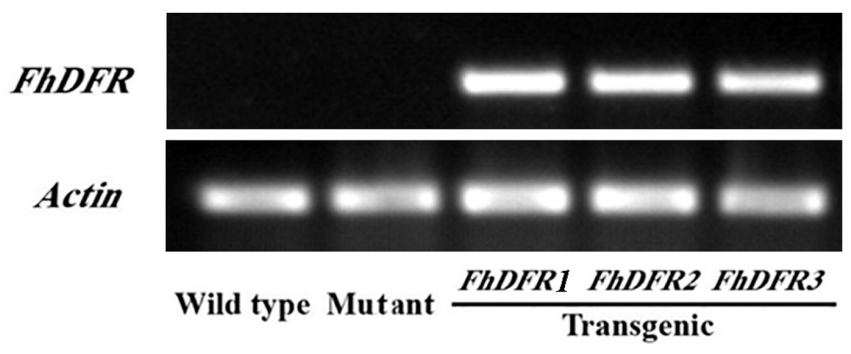

FIGURE 4 | Complementation of Arabidopsis tt3-1 mutants overexpressing FhDFR1, FhDFR2, and FhDFR3. (A) Phenotype of the wild type (WT, Ler), tt3-1 mutant and T2 transgenic lines seed coats, cotyledons and hypocotyls. (B) Expressional analysis of FhDFR1, FhDFR2, and FhDFR3 by reverse transcription polymerase chain reaction in the wild-type, tt3 mutant and transgenic lines.

fully opened flowers (Sun et al., 2015, 2016; Li et al., 2016). Eight different tissues were also collected as follows: root, leaf, scape, torus, calyx, petal, stamen, and pistil.

Similar expression patterns of FhDFR genes were observed among the five flower development stages. The expression of FhDFR1/FhDFR2 initiated from flower buds with non-pigmented tepals (Stage 1), increased gradually with the development of flowers and peaked when the flowers fully opened (Stage 5), showing an expression pattern synchronous to the anthocyanin accumulation. Moreover, FhDFR3 was also highly expressed in late stage of flower development process, which might also be involved in the biosynthesis of anthocyanins. Totally, the expression level of FhDFR3 was significantly higher than FhDFR1/FhDFR2 (Figure 3C). As for the expression patterns of the FhDFR genes in various plant tissues, quantitative realtime PCR analysis showed that the expression level of FhDFR3 transcripts was also significantly higher than FhDFR1/FhDFR2 in tested tissues (Figure 3D). However, FhDFR3 was dominantly expressed in the proanthocyanidin accumulated tissues, i.e., torus and calyx and anthocyanin accumulated tissues, petal and pistil (Li et al., 2016). In contrast, the abundant expression of FhDFR1/FhDFR2 was only observed in petal. Thus, it can be deduced that FhDFR3 fulfills important roles in the biosynthesis of both anthocyanins and proanthocyanidins in different plant tissues, and FhDFR1/FhDFR2 might be mainly responsible for the petal pigmentation. However, it is not always clear if their functions are partially or completely redundant given that the expression level of FhDFR3 was higher than FhDFR1/FhDFR2 in all the tested tissues (Figure 3D). In conclusion, the duplicated FhDFR genes might function divergently in the biosynthesis of flavonoids in F. hybrida.

\section{FhDFR1, FhDFR2, and FhDFR3 Could Complement the Arabidopsis tt3-1 Mutants}

As mentioned above, FhDFR genes showed distinct expression patterns and correlations with the accumulation of anthocyanins and proanthocynidins. In order to investigate their potential roles in the biosynthesis of flavonoids in planta, the three FhDFR genes under the control of $35 S$ promoter were introduced into the Arabidopsis mutant (tt3-1), which failed to accumulate anthocyanin pigments in their cotyledon or hypocotyls and brown tannins in their seed coats (Figure 4A). After kanamycin selection, seeds of the wild-type, Arabidopsis mutant, and T2 transgenic lines were germinated and grown on 1/2 MS medium 


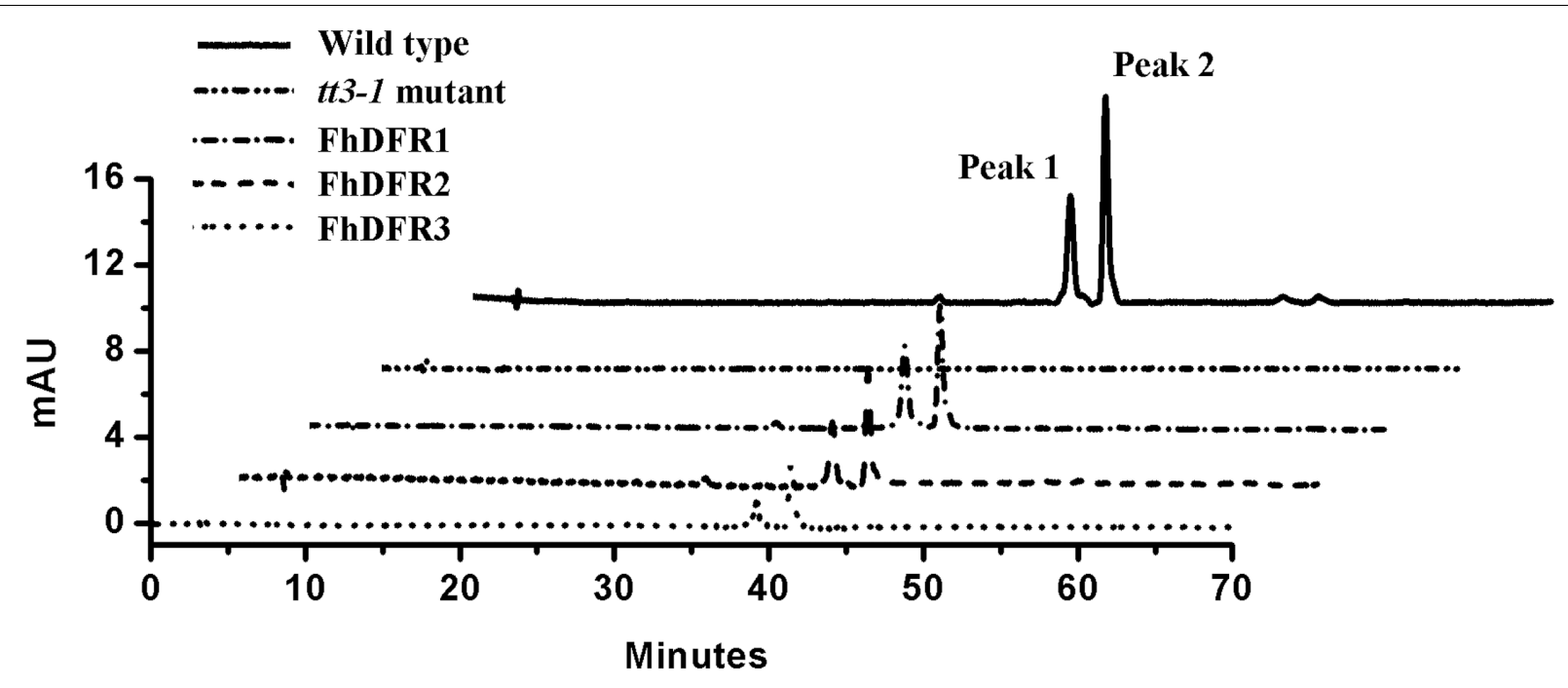

FIGURE 5 | High performance liquid chromatography analysis of anthocyanins in wild type, tt3-1 mutant and Arabidopsis transgenic seedlings. The contents of anthocyanins were measured at $520 \mathrm{~nm}$ absorbance. Peaks 1 and 2 tended to be cyanidin derivatives, cyanidin

3-O-[2-O(2-O-(sinapoyl)- $\beta$-D-xylopyranosyl)-6-O-(4-O-( $\beta$-D-glucopyranosyl)- $p$-coumaroyl)- $\beta$-D-glucopyranoside]5-O-[6-O(malonyl) $\beta$-D-glucopyranoside]methyl ester and cyanidin 3-O-[2-O(2-O-(sinapoyl)- $\beta$-D-xylopyranosyl)-6-O-(4-O-( $\beta$-D-glucopyranosyl)- $p$-coumaroyl)- $\beta$-D-glucopyranoside]5-O-[6-O(malonyl) $\beta$-D-glucopyranoside], respectively.

containing 3\% sucrose. Phenotypic observation showed that transgenic plants expressing FhDFR1, FhDFR2, and FhDFR3 genes restored the pigmentation of their seed coats and purple coloration in the cotyledons and hypocotyls (Figure 4A), whereas the mutant transformed with the empty vector were green (not shown). The transgenic lines were further confirmed for the presence and expression of exogenous genes through RT-PCR (Figure 4B). No amplicons were observed in wild type plants and mutants, whereas amplicons of expected size were observed in transgenic lines.

Furthermore, 1-week-old T2 seedlings cultured on 1/2 MS medium with $3 \% \mathrm{w} / \mathrm{v}$ sucrose were extracted and analyzed by HPLC to determine the amounts of individual anthocyanins and flavonols. The results showed that both wild type and transgenic seedlings (expressing FhDFR1, FhDFR2, FhDFR3, respectively) had two primary anthocyanin peaks compared to tt3-1 mutant seedlings which showed no relative peaks (Figure 5). Based on HPLC retention time and MS spectra with authentic compounds, these peaks were identified as cyanidin derivatives (Supplementary Table S3). As for flavonols, no difference in the peak pattern or peak height was observed in wild type plants, mutants or transgenic lines (Supplementary Figure S2). Taken together, these results demonstrated that proteins encoded by FhDFR1, FhDFR2, and FhDFR3 genes could catalyze the NADPH-dependent reduction of dihydroflavonols to leucoanthocyanidins in planta.

\section{Functional Expression in E. coli and In vitro Catalytic Activities of FhDFR Proteins}

To further confirm the enzymatic properties of FhDFR1, FhDFR2 and FhDFR3 and their potential roles in the biosynthesis of anthocyanins in F. hybrida, substrate specificity studies for FhDFR proteins with a range of substrates were tested, including DHK, DHQ, and DHM. Before catalytic assay, recombinant proteins were prepared and purified. Subsequently, the purified proteins were subjected to the biochemical analysis using DHK, DHQ, or DHM as substrate in the presence of NADPH, respectively, and the reaction products were analyzed by HPLC in comparison to authentic standards, relative retention time and UV spectra (Cheng et al., 2013). As shown in Figure 6, formation of leucodelphinidin (LEUD) was observed when using DHM as substrate in the in vitro reaction system with FhDFR1, FhDFR2, or FhDFR3 proteins, whereas only FhDFR2 could convert DHQ to LEUC because of relative lower catalytic efficiencies. On the contrary, no LEUP were observed, indicating that FhDFR1, FhDFR2, or FhDFR3 protein might not utilize DHK as substrate. These results revealed that FhDFR1, FhDFR2, and FhDFR3 performed preferentially on DHM in vitro as dihydroflavonol 4-reductases.

\section{FhDFR1/FhDFR2 and FhDFR3 Could Be Regulated by AtPAP1 and FhbHLHs}

In our recent study, two IIIf Clade-bHLH regulator genes, FhGL3L and FhTT8L, participating in anthocyanin and proanthocyanidin accumulation were isolated from F. hybrida, and Arabidopsis protoplast transfection assay demonstrated that both of them could activate the expression of AtDFR in combination with AtPAP1 ( $\mathrm{Li}$ et al., 2016). In order to verify whether the expression of FhDFR1, FhDFR2, and FhDFR3 genes were also controlled by the MBW complex (AtPAP1-FhbHLHsAtTTG1) responsible for the anthocyanin biosynthesis, target promoters of FhDFR1/FhDFR2 and FhDFR3 were firstly isolated. 


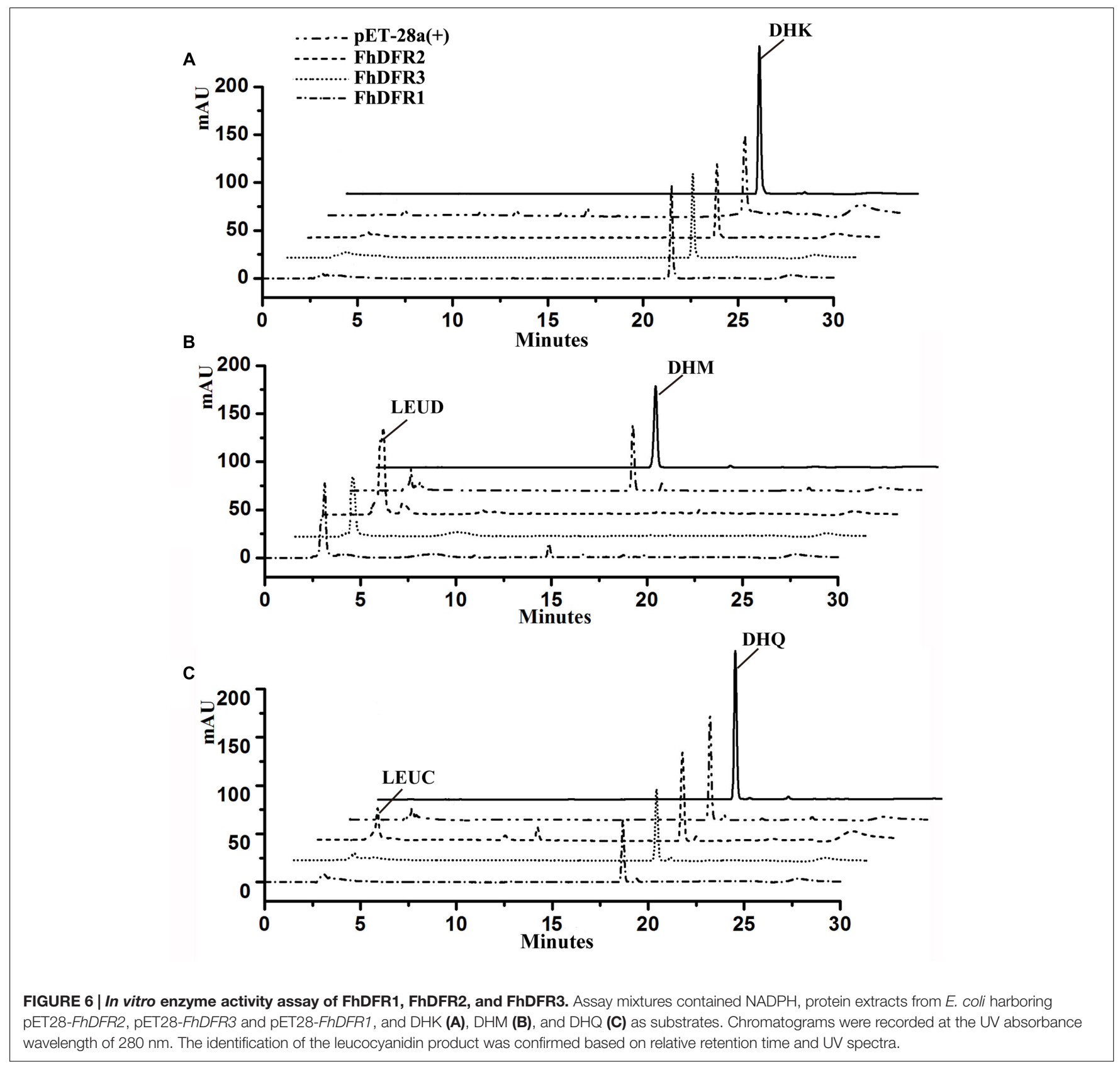

Subsequently, two bHLH regulators, i.e., FhTT8L and FhGL3L and the MYB regulator AtPAP1 were independently or cotransfected with the modified pUC19-GUS constructs, which contained the target promoters (FhDFR1/FhDFR2 and FhDFR3) driving the expression of the GUS reporter gene. As expected, both FhDFR1/FhDFR2 and FhDFR3 could be activated by FhGL3L in combination with the MYB protein AtPAP1, whereas FhTT8L could only regulate FhDFR3 in the presence of AtPAP1. The results here indicated that FhDFR1/FhDFR2 and FhDFR3 might be controlled by the MBW complex participated in flavonoid accumulation in F. hybrida (Figure 7). However, the freesia endogenous MYB regulators still need to be confirmed in the future.

\section{DISCUSSION}

Dihydroflavonol-4-reductase (DFR) genes presented strong sequence conservation in plants. In flavonoid biosynthesis, DFR is a member of the short chain dehydrogenase/reductase (SDR) superfamily which contained a highly conserved NADP-binding domain (Johnson et al., 1999, 2001; Martens et al., 2002). In this study, we firstly cloned eight putative homologous cDNA sequences of NADPH-dependent reductase genes in F. hybrida. Interestingly, FhDFR1, FhDFR2, and FhDFR3 tended to be DFR-like proteins, whereas other five proteins were more closely related to CCRs through the manual BLASTX search (Supplementary Table S1). Furthermore, FhDFR1 and FhDFR2 

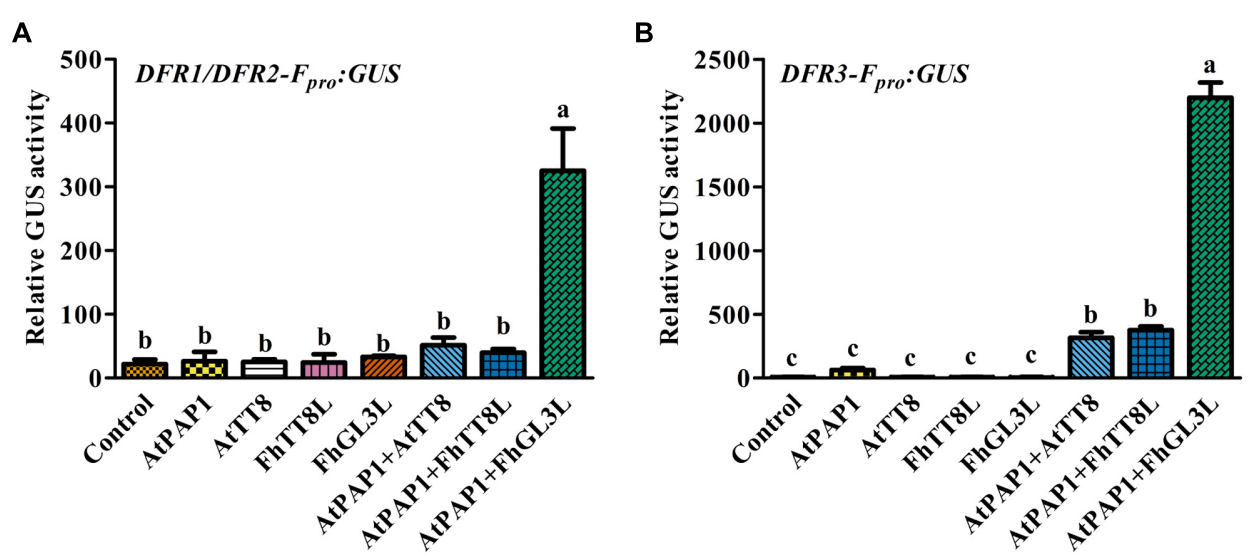

FIGURE 7 | Promoter activation of FhDFR1/FhDFR2 and FhDFR3 through Arabidopsis protoplasts transient assays. FhGL3L and FhTT8L interacted with AtPAP1 to activate FhDFR1/FhDFR2 (A) and FhDFR3 (B). Different construct combinations were transfected into Arabidopsis protoplasts. The promoter activation abilities were quantified by relative GUS activities. Data represented the mean \pm SD of three replicates. Constructs were diagrammed at the bottom of the figure. One-way ANOVA was carried out to compare statistical differences (Ducan, $p<0.05$ ).

showed high similarities in both nucleotide and amino acid sequences. cDNAs synthesized from total RNA of several tissues were used to evaluate the ratio between FhDFR1 and FhDFR2. Primer sets binding to the same site of FhDFR1 and FhDFR2 were used and at least 30 clones were sequenced. Results showed the ratio between FhDFR1 and FhDFR2 was about 1:1. Bioinformatic analysis found that the proteins encoded by these two genes as well as FhDFR3 had typical functional domains of DFR proteins, including a conserved NADPH binding motif "VTGAAGFIGSWLIMRLLERGY", a substrate specificity selective domain and several specific loci of the conservative short-chain dehydrogenase/reductase family (Johnson et al., 1999; Martens et al., 2002). In order to further investigate the potential functions of DFR-like genes in F. hybrida, we generated a phylogenic tree containing the other characterized NADPdependent reductases such as DFR, CCR, ANR, LAR, and FNR proteins in other species. As shown in Figure 2, both the amino acid alignment and phylogenic tree revealed a higher similarity between FhDFR1, FhDFR2, FhDFR3 and other plants originated and characterized DFRs (Figure 2), indicating their potential "DFR-like" catalytic properties. Furthermore, FhDFR1, FhDFR2, and FhDFR3 phylogenetically clustered into the same subgroup with DFRs from other monocot plants. Thus, it can be deduced that the divergence of DFRs most likely occurred after the division of monocots and dicots, and that there might be homologous DFR genes in different plants (Liew et al., 1998).

Previous studies demonstrated that substrate specificity of DFR could be influenced by a presumed substrate-binding region composed of 26 amino acid residues, especially the single amino acid residue at about residue 134 (Johnson et al., 2001). Consequently, DFRs could be divided into three types according to differences at this position, i.e., Asn-type DFRs, Asp-type DFRs, and non-Asn/Asp-type DFRs, the amino acid residue of the 134 position was asparagine residue (Asn), aspartic acid (Asp) and neither Asn nor Asp, respectively (Johnson et al., 2001). Generally, Asn-type DFRs could utilize all the three dihydroflavonols, DHK, DHQ, and DHM, as substrates, while Asp-type DFRs could not catalyze DHK efficiently (Forkmann and Ruhnau, 1987; Helariutta et al., 1993; Tanaka et al., 1995; Johnson et al., 1999). However, FhDFR1 and FhDFR2 belonged to Asn-type DFRs, no pelargonidin was detected in flowers of F. hybrida, and no LEUP was detected in the in vitro catalytic activity assays, which was consistent with ApDFR1 in Agapanthus praecox ssp.orientalis (Leighton) (Mori et al., 2014). Commonly, Asn-type DFRs are widely distributed in plants, whereas Asptype DFRs are found in limited plant species that are scattered throughout the eudicots (Shimada et al., 2005). In contrast, FhDFR3, as well as IhDFR from Iris $\times$ hollandica, broke the rule, which belonged to monocotyledonous Asp-type DFRs.

The expression patterns of FhDFR genes of F. hybrida were tested temporally and spatially. In the development process of flowers, the amount of anthocyanin increased gradually and peaked when flowers fully opened, whereas the proanthocyanidins were constitutively accumulated at a relative low level (Li et al., 2016). FhDFR1/FhDFR2 was parallel well to the anthocyanin accumulation as well as FhDFR3, which showed a high expression level at the late stage of the flower development process. In addition, both anthocyanin and proanthocyanidin accumulated more extensively in flower tissues than vegetative tissues, and petal and torus were the dominant tissues for anthocyanin and proanthocyanidin biosynthesis, respectively ( $\mathrm{Li}$ et al., 2016), which was also synchronous to expression patterns of FhDFR1/FhDFR2 and FhDFR3 in these tissues. However, the expression level of FhDFR3 was significantly higher than FhDFR1/FhDFR2 genes in all tested tissues, which might imply their partial or complete redundant roles. The spatial and temporal expression characteristics of FhDFR genes were found similar in several other species (Nakatsuka et al., 2003; Li et al., 2012; Mori et al., 2014).

In order to investigate the functional divergence of the three FhDFR genes in the flavonoid biosynthesis, FhDFR1, FhDFR2, and FhDFR3 were introduced into Arabidopsis tt3-1 mutants, and 


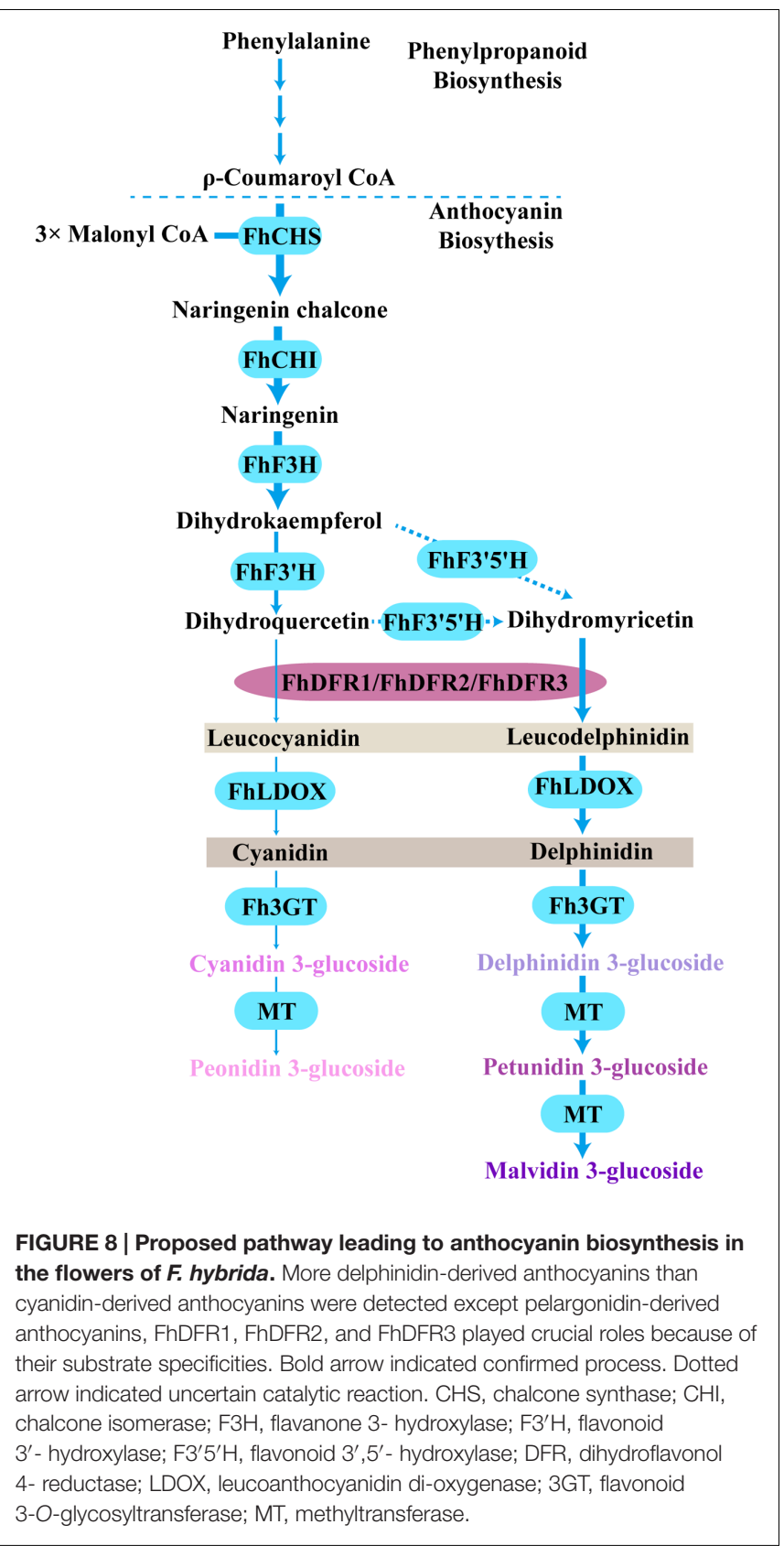

the results showed that cyanidin derivatives could be detected in the transgenic plants overexpressing exogenous FhDFR1, FhDFR2, FhDFR3 genes, indicating that these three genes could utilize DHQ as substrate in Arabidopsis. Because the contents of the cyanidin derivatives were significantly lower compared with the wild type plants, it can be deduced that FhDFR1, FhDFR2, FhDFR3 might have relative weaker catalytic efficiency for DHQ in contrast to AtDFR itself. On the other hand, no pelargonidin derivatives were detected in transgenic plants, implying that FhDFR1, FhDFR2, FhDFR3 might be deficient in the catalysis of DHK. As known, there is no DHM accumulated in Arabidopsis due to lacking of flavonoid $3^{\prime}, 5^{\prime}$-hydroxylase $\left(\mathrm{F}^{\prime} 5^{\prime} \mathrm{H}\right)$, whether FhDFR1, FhDFR2, FhDFR3 could accept DHM as substrate should be further validated by the examination of their catalytic properties. Based on the in vitro biochemical assays, we found that all the three FhDFR proteins aforementioned showed a high catalytic efficiency for DHM, whereas only FhDFR2 was proved to convert DHQ to LEUC, which was not consistent to the results of the Arabidopsis tt3-1 mutant complementation mentioned above, this contradiction might be ascribed to the lower catalytic efficiency of FhDFR1 and FhDFR3, which was lost during the preparation and purification of the recombinant proteins. In conclusion, FhDFR1, FhDFR2, and FhDFR3 could utilize both DHQ and DHM as substrate, with a higher activity toward DHM than DHQ.

Based on the metabolites found in the flowers, the anthocyanin biosynthetic pathway in freesia was proposed earlier (Sun et al., 2016). All of the six basic anthocyanin aglycons could be synthesized except pelargonidin derivatives, and the most abundant anthocyanins were delphinidin derivatives. On the contrary, flavonol analysis showed predominant kaempferol glycosides and minor quercetin glycosides, whereas myricetin glycosides were undetectable throughout the flower development (Sun et al., 2016). Therefore, as all the precursors of pelargonidin, cyanidin and delphinidin were present in F. hybrida, the lack of pelargonidin might be ascribed to the substrate specificity of the FhDFR proteins. In addition, it seemed reasonable to deduce that the lack of myricetin in F. hybrida flowers might result from the substrate selectivity of FLS (Figure 8). Thus, it was interesting to conclude that FLS and DFR competed for common substrates in order to direct the biosynthesis of flavonols and anthocyanins, respectively, which was also illustrated by Luo et al. (2015) earlier. Actually, several other plant species, such as Petunia hybrida, Cymbidium hybrida, Angelonia angustifolia, Agapanthus praecox ssp. orientalis (Leighton), have also been found unable to produce pelargonidin derivatives because of the DFR substrate specificities (Mol et al., 1998; Johnson et al., 1999; Gosch et al., 2014; Mori et al., 2014). Therefore, it can be concluded that substrate specificities of FhDFR proteins played crucial role in determination of anthocyanin aglycons in F. hybrdia.

The DFRs are key enzymes in flavonoid biosynthesis. Variable $D F R$-like gene numbers were therefore found in various genomes, e.g., a single copy $D F R$ is present in Arabidopsis thaliana, in which the anthocyanin and proanthocyanidin components seemed to be simple as well, while multicopy DFR genes exist in M. truncatula, L. japonicus, Populus trichocarpa, and F. hybrida, these metabolites showed more complicated patterns and more diverse physiological functions (Østergaard et al., 2001; Xie et al., 2004; Shimada et al., 2005; Huang et al., 2012). Susumu Ohno hypothesized that gene duplication drives the evolution of novel functions, and deduced three kind fates of the duplicated genes: silence, neofunctionalization and subfunctionalization (Epstein, 1971). Based on the phylogenetic position of DFR, ANR, FNR, and CCR, it can be deduced, that they might have diverged from the same ancestral gene after gene or genome duplication during plant evolution. It could also be expected that some DFR isozymes might be specialized to anthocyanin synthesis, proanthocyanidin or other branch pathways in the bioysnthesis of flavonoids. Huang et al. (2012) found that overexpressing PtrDFR1 in 
Chinese white poplar (Populus tomentosa Carr.) resulted in a higher accumulation of both anthocyanins and condensed tannins, whereas constitutively expressing PtrDFR2 only improved condensed tannin accumulation. In addition, the different paralogs might be regulated differentially with spatial and temporal manner under exogenous and endogenous cues. In this study, the expression of both FhDFR1/FhDFR2 and FhDFR3 was testified to be controlled by a common MBW complex, including MYB and bHLH regulators. However, both FhDFR1/FhDFR2 and FhDFR3 could be activated by FhGL3L in combination with the MYB protein AtPAP1, whereas FhTT8L could only regulate FhDFR3 in the presence of AtPAP1. The endogenous FhMYB regulators still need to be further investigated whether the phenomena simply resulting from the heterogenous AtPAP1 or from the evolutionary divergence between FhDFR1/FhDFR2 and FhDFR3.

\section{CONCLUSION}

Previous studies showed that gene duplication acted as a driver for plant morphogenetic evolution, and have possibly allowed the adaptation of the enzymes for specialized functions and contributed to the divergence of plant metabolisms (Rensing, 2014). In this study, we found that the duplicated FhDFR genes from $F$. hybrida have evolved divergently with different nucleotide sequences and expression patterns. FhDFR1, FhDFR2, and FhDFR3 were involved in the biosynthesis of flavonoinds and determined the components of the anthocyanins in F. hybrida. Comparatively speaking, FhDFR3 might perform more important roles in the biosynthesis of proanthocyanidins. Moreover, they were controlled by a potential MBW complex

\section{REFERENCES}

Baudry, A., Heim, M. A., Dubreucq, B., Caboche, M., Weisshaar, B., and Lepiniec, L. (2004). TT2, TT8, and TTG1 synergistically specify the expression of BANYULS and proanthocyanidin biosynthesis in Arabidopsis thaliana. Plant J. 39, 366-380. doi: 10.1111/j.1365-313X.2004. 02138.x

Cheng, H., Li, L., Cheng, S., Cao, F., Xu, F., Yuan, H., et al. (2013). Molecular cloning and characterization of three genes encoding dihydroflavonol-4reductase from Ginkgo biloba in anthocyanin biosynthetic pathway. PLoS ONE 8:e72017. doi: 10.1371/journal.pone.0072017

Clough, S. J., and Bent, A. F. (1998). Floral dip: a simplified method for Agrobacterium-mediated transformation of Arabidopsis thaliana. Plant J. 16, 735-743. doi: 10.1046/j.1365-313x.1998.00343.x

Davies, K. M., Schwinn, K. E., Deroles, S. C., Manson, D. G., Lewis, D. H., Bloor, S. J., et al. (2003). Enhancing anthocyanin production by altering competition for substrate between flavonol synthase and dihydroflavonol 4-reductase. Euphytica 131, 259-268. doi: 10.1023/A:1024018729349

Des Marais, D. L., and Rausher, M. D. (2008). Escape from adaptive conflict after duplication in an anthocyanin pathway gene. Nature 454, 762-765. doi: $10.1038 /$ nature 07092

Epstein, C. J. (1971). Evolution by Gene Duplication. Berlin: Springer.

Fischer, T. C., Halbwirth, H., Meisel, B., Stich, K., and Forkmann, G. (2003). Molecular cloning, substrate specificity of the functionally expressed dihydroflavonol 4-reductases from Malus domestica and Pyrus communis cultivars and the consequences for flavonoid metabolism. Arch. Biochem. Biophys. 412, 223-230. doi: 10.1016/S0003-9861(03) 00013-4 responsible for anthocyanin biosynthesis. Taken together, the results are not only helpful for future research on DFR evolution and divergence analysis but also useful for manipulating flavonoid biosynthesis in F. hybrida as well as in other monocotyledonous ornamental plants.

\section{AUTHOR CONTRIBUTIONS}

$\mathrm{YL}, \mathrm{XL}, \mathrm{XC}, \mathrm{XS}$, and RG performed most of the experiments. SY and $\mathrm{TH}$ helped in seedling planting and sample preparation, SW helped analyze the results, XG designed the experiments, wrote and edited the manuscript. LW helped design the experiments and revise the manuscript. All authors read and approved the final manuscript.

\section{FUNDING}

This work was supported by the National Natural Science Foundation of China (31170276, 31570295), the Fundamental Research Fund for the Central Universities (2412015ZH006) and the Program for Introducing Talents to Universities (B07017). The funders had no role in study design, data collection and analysis, decision to publish, or preparation of the manuscript.

\section{SUPPLEMENTARY MATERIAL}

The Supplementary Material for this article can be found online at: http://journal.frontiersin.org/article/10.3389/fpls.2017.00428/ full\#supplementary-material

Forkmann, G., and Martens, S. (2001). Metabolic engineering and applications of flavonoids. Curr. Opin. Biotechnol. 12, 155-160. doi: 10.1016/S0958-1669(00) 00192-0

Forkmann, G., and Ruhnau, B. (1987). Distinct substrate specificity of dihydroflavonol 4-reductase from flowers of Petunia hybrida. Z. Naturforsch. 42C, 1146-1148. doi: 10.1515/znc-1987-9-1026

Gonzalez, A., Zhao, M., Leavitt, J. M., and Lloyd, A. M. (2008). Regulation of the anthocyanin biosynthetic pathway by the TTG1/bHLH/Myb transcriptional complex in Arabidopsis seedlings. Plant J. 53, 814-827. doi: 10.1111/j.1365313X.2007.03373.x

Gosch, C., Nagesh, K. M., Thill, J., Miosic, S., Plaschil, S., Milosevic, M., et al. (2014). Isolation of dihydroflavonol 4-reductase cDNA clones from Angelonia $\mathrm{x}$ angustifolia and heterologous expression as GST fusion protein in Escherichia coli. PLoS ONE 9:e107755. doi: 10.1371/journal.pone.0107755

Halbwirth, H., Kahl, S., Jäger, W., Reznicek, G., Forkmann, G., and Stich, K. (2006). Synthesis of (C-14)-labeled 5-deoxyflavonoids and their application in the study of dihydroflavonol/leucoanthocyanidin interconversion by dihydroflavonol 4reductase. Plant Sci. 170, 587-595. doi: 10.1016/j.plantsci.2005.10.013

Halbwirth, H., Martens, S., Wienand, U., Forkmann, G., and Stich, K. (2003). Biochemical formation of anthocyanins in silk tissue of Zea mays. Plant Sci. 164, 489-495. doi: 10.1016/S0168-9452(02)00433-8

Helariutta, Y., Elomaa, P., Kotilainen, M., Seppänen, P., and Teeri, T. H. (1993). Cloning of cDNA coding for dihydroflavonol-4-reductase (DFR) and characterization of $d f r$ expression in the corollas of Gerbera hybrida var. Regina (Compositae). Plant Mol. Biol. 22, 183-193. doi: 10.1007/BF00014927

Hichri, I., Barrieu, F., Bogs, J., Kappel, C., Delrot, S., and Lauvergeat, V. (2011). Recent advances in the transcriptional regulation of the flavonoid biosynthetic pathway. J. Exp. Bot. 62, 2465-2483. doi: 10.1093/jxb/erq442 
Holton, T. A., and Cornish, E. C. (1995). Genetics and biochemistry of anthocyanin biosynthesis. Plant Cell 7, 1071-1083. doi: 10.1105/tpc.7.7.1071

Huang, Y., Gou, J., Jia, Z., Yang, L., Sun, Y., Xiao, X., et al. (2012). Molecular cloning and characterization of two genes encoding dihydroflavonol-4-reductase from Populus trichocarpa. PLoS ONE 7:e30364. doi: 10.1371/journal.pone.0030364

Jaakola, L. (2013). New insights into the regulation of anthocyanin biosynthesis in fruits. Trends Plant Sci. 18, 477-483. doi: 10.1016/j.tplants.2013.06.003

Johnson, E. T., Ryu, S., Yi, H., Shin, B., Cheong, H., and Choi, G. (2001). Alteration of a single amino acid changes the substrate specificity of dihydroflavonol 4-reductase. Plant J. 25, 325-333. doi: 10.1046/j.1365-313x.2001.00962.x

Johnson, E. T., Yi, H., Shin, B., Oh, B. J., Cheong, H., and Choi, G. (1999). Cymbidium hybrida dihydroflavonol 4-reductase does not efficiently reduce dihydrokaempferol to produce orange pelargonidin-type anthocyanins. Plant J. 19, 81-85. doi: 10.1046/j.1365-313X.1999.00502.x

Katsumoto, Y., Fukuchimizutani, M., Fukui, Y., Brugliera, F., Holton, T. A., Karan, M., et al. (2007). Engineering of the rose flavonoid biosynthetic pathway successfully generated blue-hued flowers accumulating delphinidin. Plant Cell Physiol. 48, 1589-1600. doi: 10.1093/pcp/pcm131

Kawahigashi, H., Kasuga, S., Sawada, Y., Yonemaru, J., Ando, T., Kanamori, H., et al. (2016). The Sorghum gene for leaf color changes upon wounding $(P)$ encodes a flavanone 4-reductase in the 3-deoxyanthocyanidin biosynthesis pathway. G3 6, 1439-1447. doi: 10.1534/g3.115.026104/-/DC1

Lacombe, E., Hawkins, S., Doorsselaere, J. V., Piquemal, J., Goffner, D., Poeydomenge, O., et al. (1997). Cinnamoyl CoA reductase, the first committed enzyme of the lignin branch biosynthetic pathway: cloning, expression and phylogenetic relationships. Plant J. 11, 429-441. doi: 10.1046/j.1365-313X.1997. 11030429.x

Li, H., Qiu, J., Chen, F., Lv, X., Fu, C., Zhao, D., et al. (2012). Molecular characterization and expression analysis of dihydroflavonol 4-reductase (DFR) gene in Saussurea medusa. Mol. Biol. Rep. 39, 2991-2999. doi: 10.1007/s11033011-1061-2

Li, Y., Shan, X., Gao, R., Yang, S., Wang, S., Gao, X., et al. (2016). Two IIIf cladebHLHs from Freesia hybrida play divergent roles in flavonoid biosynthesis and trichome formation when ectopically expressed in Arabidopsis. Sci. Rep. 6:30514. doi: 10.1038/srep30514

Liew, C. F., Loh, C. S., Goh, C. J., and Lim, S. H. (1998). The isolation, molecular characterization and expression of dihydroflavonol 4-reductase cDNA in the orchid, Bromheadia finlaysoniana. Plant Sci. 135, 161-169. doi: 10.1016/s01689452(98)00071-5

Liu, H., Du, Y., Chu, H., Shih, C. H., Wong, Y. W., Wang, M., et al. (2010). Molecular dissection of the pathogen-inducible 3-deoxyanthocyanidin biosynthesis pathway in sorghum. Plant Cell Physiol. 51, 1173-1185. doi: $10.1093 /$ pcp/pcq080

Livak, K. J., and Schmittgen, T. D. (2001). Analysis of relative gene expression data using real-time quantitative PCR and the $2-\Delta \Delta \mathrm{C}$ T method. Methods 25, 402-408. doi: 10.1006/meth.2001.1262

Lo, S. C., and Nicholson, R. L. (1998). Reduction of light-induced anthocyanin accumulation in inoculated sorghum mesocotyls. Implications for a compensatory role in the defense response. Plant Physiol. 116, 979-989. doi: 10.1104/pp.116.3.979

Luo, P., Ning, G., Wang, Z., Shen, Y., Jin, H., Li, P., et al. (2015). Disequilibrium of flavonol synthase and dihydroflavonol-4-reductase expression associated tightly to white vs. red color flower formation in plants. Front. Plant Sci. 6:1257. doi: 10.3389/fpls.2015.01257

Martens, S., Preuss, A., and Matern, U. (2010). ChemInform abstract: multifunctional flavonoid dioxygenases: flavonol and anthocyanin biosynthesis in Arabidopsis thaliana L. Phytochemistry 71, 1040-1049. doi: 10.1016/j. phytochem.2010.04.016

Martens, S., Teeri, T., and Forkmann, G. (2002). Heterologous expression of dihydroflavonol 4-reductases from various plants. FEBS Lett. 531, 453-458. doi: 10.1016/S0014-5793(02)03583-4

Miller, R., Owens, S. J., and Rørslett, B. (2011). Plants and colour: flowers and pollination. Opt. Laser. Technol. 43, 282-294. doi: 10.1016/j.optlastec.2008. 12.018

Mol, J., Grotewold, E., and Koes, R. (1998). How gens paint flowers and seeds. Trends Plant Sci. 3, 212-217. doi: 10.1016/S1360-1385(98)01242-4

Mori, S., Otani, M., Kobayashi, H., and Nakano, M. (2014). Isolation and characterization of the dihydroflavonol 4-reductase gene in the monocotyledonous ornamental Agapanthus praecox ssp. orientalis (Leighton) Leighton. Sci. Hortic. 166, 1-8. doi: 10.1016/j.scienta.2013.12.009

Morita, Y., Takagi, K., Fukuchi-Mizutani, M., Ishiguro, K., Tanaka, Y., Nitasaka, E., et al. (2014). A chalcone isomerase-like protein enhances flavonoid production and flower pigmentation. Plant J. 78, 294-304. doi: 10.1111/tpj.12469

Murashige, T., and Skoog, F. (1962). A revised medium for rapid growth and bio assays with tobacco tissue cultures. Physiol. Plant. 15, 473-497. doi: 10.1111/j. 1399-3054.1962.tb08052.x

Nakatsuka, A., Izumi, Y., and Yamagishi, M. (2003). Spatial and temporal expression of chalcone synthase and dihydroflavonol 4-reductase genes in the Asiatic hybrid lily. Plant Sci. 165, 759-767. doi: 10.1016/S0168-9452(03)00 254-1

Østergaard, L., Lauvergeat, V., Næsted, H., Mattsson, O., and Mundy, J. (2001). Two differentially regulated Arabidopsis genes define a new branch of the DFR superfamily. Plant Sci. 160, 463-472. doi: 10.1016/S0168-9452(00)00407-6

Patra, B., Schluttenhofer, C., Wu, Y., Pattanaik, S., and Yuan, L. (2013). Transcriptional regulation of secondary metabolite biosynthesis in plants. Biochim. Biophys. Acta 1829, 1236-1247. doi: 10.1016/j.bbagrm.2013. 09.006

Penninckx, I. A., Eggermont, K., Terras, F. R., Thomma, B. P., Samblanx, G. W., Buchala, A., et al. (1997). Pathogen-induced systemic activation of a plant defensin gene in Arabidopsis follows a salicylic acid-independent pathway. Plant Cell 8, 2309-2323. doi: 10.2307/3870470

Petit, P., Granier, T., D’Estaintot, B. L., Manigand, C., Bathany, K., Schmitter, J. M., et al. (2007). Crystal structure of grape dihydroflavonol 4-reductase, a key enzyme in flavonoid biosynthesis. J. Mol. Biol. 368, 1345-1357. doi: 10.1016/j. jmb.2007.02.088

Petroni, K., and Tonelli, C. (2011). Recent advances on the regulation of anthocyanin synthesis in reproductive organs. Plant Sci. 181, 219-229. doi: 10.1016/j.plantsci.2011.05.009

Piero, A. R. L., Puglisi, I., and Petrone, G. (2006). Gene characterization, analysis of expression and in vitro synthesis of dihydroflavonol 4-reductase from [Citrus sinensis (L.) Osbeck]. Phytochemistry 67, 684-695. doi: 10.1016/j.phytochem. 2006.01.025

Pires, N., and Dolan, L. (2009). Origin and diversification of basic-helix-loop-helix proteins in plants. Mol. Biol. Evol. 27, 862-874. doi: 10.1093/molbev/msp288

Rensing, S. A. (2014). Gene duplication as a driver of plant morphogenetic evolution. Curr. Opin. Plant Biol. 17, 43-48. doi: 10.1016/j.pbi.2013.11.002

Shih, C.-H., Chu, I. K., Yip, W. K., and Lo, C. (2006). Differential expression of two flavonoid 3'-hydroxylase cDNAs involved in biosynthesis of anthocyanin pigments and 3-deoxyanthocyanidin phytoalexins in sorghum. Plant Cell Physiol. 47, 1412-1419. doi: 10.1093/pcp/pcl003

Shimada, N., Sasaki, R., Sato, S., Kaneko, T., Tabata, S., Aoki, T., et al. (2005). A comprehensive analysis of six dihydroflavonol 4-reductases encoded by a gene cluster of the Lotus japonicus genome. J. Exp. Bot. 56, 2573-2585. doi: 10.1093/jxb/eri251

Shirley, B. W., Hanley, S., and Goodman, H. M. (1992). Effects of ionizing radiation on a plant genome: analysis of two Arabidopsis transparent testa mutations. Plant Cell 4, 333-347. doi: 10.1105/tpc.4.3.333

Sievers, F., Wilm, A., Dineen, D., Gibson, T. J., Karplus, K., Li, W., et al. (2011). Fast, scalable generation of high-quality protein multiple sequence alignments using Clustal Omega. Mol. Syst. Biol. 7:539. doi: 10.1038/msb. 2011.75

Singh, K., Kumar, S., Yadav, S. K., and Ahuja, P. S. (2009). Characterization of dihydroflavonol 4-reductase cDNA in tea [Camellia sinensis (L.) O. Kuntze]. Plant Biotechnol. Rep. 3, 95-101. doi: 10.1007/s11816-008-0079-y

Smith, S. D., and Rausher, M. D. (2011). Gene loss and parallel evolution contribute to species difference in flower color. Mol. Biol. Evol. 28, 2799-2810. doi: 10.1093/ molbev/msr109

Smith, S. D., Wang, S., and Rausher, M. D. (2013). Functional evolution of an anthocyanin pathway enzyme during a flower color transition. Mol. Biol. Evol. 30, 602-612. doi: 10.1093/molbev/mss 255

Styles, E. D., and Ceska, O. C. (1975). Genetic control of 3-hydroxy- and 3deoxyflavonoids in Zea mays. Phytochemistry 14, 413-415. doi: 10.1016/00319422(75)85101-6

Sui, X., Gao, X., Ao, M., Wang, Q., Yang, D., Wang, M., et al. (2011). cDNA cloning and characterization of UDP-glucose: anthocyanidin 3-O-glucosyltransferase in Freesia hybrida. Plant Cell Rep. 30, 1209-1218. doi: 10.1007/s00299-011-1029-7 
Sun, W., Liang, L., Meng, X., Li, Y., Gao, F., Liu, X., et al. (2016). Biochemical and molecular characterization of a flavonoid 3-O-glycosyltransferase responsible for anthocyanins and flavonols biosynthesis in Freesia hybrida. Front. Plant Sci. 7:410. doi: 10.3389/fpls.2016.00410

Sun, W., Meng, X., Liang, L., Jiang, W., Huang, Y., He, J., et al. (2015). Molecular and biochemical analysis of chalcone synthase from Freesia hybrid in flavonoid biosynthetic pathway. PLoS ONE 10:e0119054. doi: 10.1371/journal. pone.0119054

Tamura, K., Stecher, G., Peterson, D., Filipski, A., and Kumar, S. (2013). MEGA6: molecular evolutionary genetics analysis version 6.0. Mol. Biol. Evol. 30, 2725-2729. doi: 10.1093/molbev/mst197

Tanaka, Y., Fukui, Y., Fukuchi-Mizutani, M., Holton, T. A., Higgins, E., and Kusumi, T. (1995). Molecular cloning and characterization of Rosa hybrida dihydroflavonol 4-reductase gene. Plant Cell Physiol. 36, 1023-1031. doi: 10.1093/oxfordjournals.pcp.a078844

Tanaka, Y., Sasaki, N., and Ohmiya, A. (2008). Biosynthesis of plant pigments: anthocyanins, betalains and carotenoids. Plant J. 54, 733-749. doi: 10.1111/j. 1365-313X.2008.03447.x

Wang, H., Fan, W., Li, H., Yang, J., Huang, J., and Zhang, P. (2013). Functional characterization of Dihydroflavonol-4-reductase in anthocyanin biosynthesis of purple sweet potato underlies the direct evidence of anthocyanins function against abiotic stresses. PLOS ONE 8:e78484. doi: 10.1371/journal.pone.007 8484

Wang, S., and Chen, J. G. (2014). Regulation of cell fate determination by singlerepeat R3 MYB transcription factors in Arabidopsis. Front. Plant Sci. 5:133. doi: 10.3389/fpls.2014.00133

Xie, D. Y., and Dixon, R. A. (2005). Proanthocyanidin biosynthesis-still more questions than answers? Phytochemistry 66, 2127-2144. doi: 10.1016/j. phytochem.2005.01.008

Xie, D. Y., Jackson, L. A., Cooper, J. D., Ferreira, D., and Paiva, N. L. (2004). Molecular and biochemical analysis of two cDNA clones encoding dihydroflavonol-4-reductase from Medicago truncatula. Plant Physiol. 134, 979-994. doi: 10.1104/pp.103.030221
Xie, D. Y., Sharma, S. B., Paiva, N. L., Ferreira, D., and Dixon, R. A. (2003). Role of anthocyanidin reductase, encoded by BANYULS in plant flavonoid biosynthesis. Science 299, 396-399. doi: 10.1126/science.1078540

Xu, W., Dubos, C., and Lepiniec, L. (2015). Transcriptional control of flavonoid biosynthesis by MYB-bHLH-WDR complexes. Trends Plant Sci. 20, 176-185. doi: 10.1016/j.tplants.2014.12.001

Xu, W., Grain, D., Bobet, S., Gourrierec, J. L., Thévenin, J., Kelemen, Z., et al. (2014). Complexity and robustness of the flavonoid transcriptional regulatory network revealed by comprehensive analyses of MYB-bHLH-WDR complexes and their targets in Arabidopsis seed. New Phytol. 202, 132-144. doi: 10.1111/ nph. 12620

Xue, S., Valdez, D., Collman, P. I., and Diamant, N. E. (2011). Recent advances on the regulation of anthocyanin synthesis in reproductive organs. Plant Sci. 181, 219-229. doi: 10.1016/j.plantsci.2011.05.009

Yoshida, K., Iwasaka, R., Shimada, N., Ayabe, S., Aoki, T., and Sakuta, M. (2010). Transcriptional control of the dihydroflavonol 4-reductase multigene family in Lotus japonicus. J. Plant Res. 123, 801-805. doi: 10.1007/s10265-010-0325-6

Zhou, L., Zheng, K., Wang, X., Tian, H., Wang, X., and Wang, S. (2014). Control of trichome formation in Arabidopsis by poplar single-repeat R3 MYB transcription factors. Front. Plant Sci. 5:262. doi: 10.3389/fpls.2014. 00262

Conflict of Interest Statement: The authors declare that the research was conducted in the absence of any commercial or financial relationships that could be construed as a potential conflict of interest.

Copyright (c) 2017 Li, Liu, Cai, Shan, Gao, Yang, Han, Wang, Wang and Gao. This is an open-access article distributed under the terms of the Creative Commons Attribution License (CC BY). The use, distribution or reproduction in other forums is permitted, provided the original author(s) or licensor are credited and that the original publication in this journal is cited, in accordance with accepted academic practice. No use, distribution or reproduction is permitted which does not comply with these terms. 